\title{
Hydride Vapour Phase Homoepitaxial Growth of GaN on MOCVD-Grown 'Templates'
}

\author{
T. Paskova ${ }^{1}$, S. Tungasmita ${ }^{1}$, E. Valcheva ${ }^{1}$, E.B. Svedberg ${ }^{1}$, B. Arnaudov ${ }^{2}$, \\ S. Evtimova ${ }^{2}$, P.Å. Persson ${ }^{1}$, A. Henry ${ }^{1}$ R. Beccard ${ }^{3}$, M. Heuken ${ }^{3}$ and B. Monemar ${ }^{1}$ \\ ${ }^{1}$ IFM, Linköping University, S-581 83 Linköping, Sweden \\ ${ }^{2}$ Faculty of Physics, Sofia University, 5, J. Bourchier blvd., Sofia 1164, Bulgaria \\ ${ }^{3}$ Aixtron AG, D-52072 Aachen, Germany
}

\begin{abstract}
We report on an improved quality of thick HVPE-GaN grown on MOCVD-GaN 'template' layers compared to the material grown directly on sapphire. The film-substrate interface revealed by cathodoluminescence measurements shows an absence of highly doped columnar structures which are typically present in thick HVPE-GaN films grown directly on sapphire. This improved structure results in a reduction of two orders of magnitude of the free carrier concentration from Hall measurements. It was found that the structure, morphology, electrical and optical properties of homoepitaxial thick GaN layers grown by HVPE were strongly influenced by the properties of the MOCVD-GaN 'template'. Additionally the effect of Si doping of the GaN buffer layers on the HVPE$\mathrm{GaN}$ properties was analysed.
\end{abstract}

\section{INTRODUCTION}

Over the last years considerable improvements have been made in the crystalline quality of thick GaN layers grown by HVPE [1,2] although many defect related issues of thick films are still rather controversial. These materials still have a high-density of threading dislocations and stacking mismatch boundaries due to heteroepitaxial mismatch with the most commonly used sapphire substrates.

The development of pre-growth processes in MOCVD growth, involving either a sapphire nitridation or $\mathrm{GaCl}$ pretreatment, or using different buffer layers, results in optimisation of nucleation steps, which play a dominant role in determining the properties of subsequently grown layers. Using low temperature AIN or GaN buffers, the MOCVD growth successfully overcomes many drawbacks as cracks and high residual concentration [3], however, the same approach was found to result in poor quality material in HVPE growth. Promising results for the combination of MOCVD-grown GaN 'templates' with subsequent homoepitaxially grown $\mathrm{GaN}$ by molecular beam epitaxy, have been recently reported [4]. At the moment, only a $\mathrm{ZnO}$ buffer has been reported to be a suitable buffer for growth of thick HVPE-GaN layers, even though it is largely desorbed at the early stages of growth [1].

In this study, we concentrate on MOCVD-GaN 'template' layers as an alternative buffer for GaN growth by HVPE. The material properties of the HVPE-GaN layers have been investigated by different techniques, and their relationship with the buffer layer properties has been exposed.

\section{EXPERIMENTAL}

The GaN layers were grown in a conventional HVPE system described previously [5] on c-plane sapphire substrates. To ensure a better nucleation of the GaN, we utilised 
MOCVD-GaN 'template' layers with a thickness of $2.5 \mu \mathrm{m}$ grown in the Aixtron application laboratory. The MOCVD growth was carried out at $1170{ }^{\circ} \mathrm{C}$. Two types of MOCVD 'templates', intentionally doped with $\mathrm{Si}$ with a free carrier concentration of $1.8 \times 10^{18} \mathrm{~cm}^{-3}$ and intentionally undoped GaN layers with a free carrier concentration of $2 \times 10^{17} \mathrm{~cm}^{-3}$ were used. The HVPE layers were grown at $1090{ }^{\circ} \mathrm{C} . \mathrm{NH}_{3}, \mathrm{Ga}$ and $\mathrm{HCl}$ were used as source materials under a stream of $\mathrm{N}_{2}$ carrier gas. The substrates with MOCVD$\mathrm{GaN}$ buffers were heated up to the growth temperature in a $40 \% \mathrm{NH}_{3}$ atmosphere in order to protect the buffers. A layer without a buffer was grown using a nitridation pretreatment of the sapphire in $20 \% \mathrm{NH}_{3}$ for 15 minutes at the growth temperature, in the following referred to as a reference sample. High growth rates of $95-110 \mu \mathrm{m} / \mathrm{h}$ were used, and the thicknesses of the layers studied in this work were in the range of $22-25 \mu \mathrm{m}$.

The material properties of both MOCVD-GaN buffers and HVPE-GaN layers were investigated by a variety of techniques as x-ray diffraction (XRD) using a triple axis configuration providing a resolution of $\sim 10 \mathrm{arcsec}$; atomic force microscopy (AFM) using a Nanoscope IIIa instrument operated in tapping mode; transmission electron microscopy (TEM); 7K cathotoluminescence (CL) using a Gemini 1550 LEO electron microscope with an accelerating voltage of $15 \mathrm{kV} ; 2 \mathrm{~K}$ photoluminescence (PL) using the $244 \mathrm{~nm}$ wavelength of a frequency-doubled Ar ion laser; and variable temperature Hall effect measurements ( $\mathrm{T}=80-300 \mathrm{~K}$ ) using the Van-der-Pauw method.

\section{RESULTS AND DISCUSSION}

Doping of the MOCVD-GaN by Si to a concentration of $(3-8) \times 10^{18} \mathrm{~cm}^{-3}$ has been reported to reduce the biaxial stress in the layers as well as to reduce the density of vertical screw and edge dislocations along the c-axis [6,7]. Simultaneously, the density of misfit dislocations parallel to the interface has increased [7]. Such structural peculiarities have been found to promote an increase in mobility in the Si-doped epilayers [7]. That is why we investigate both type of MOCVD 'templates', slightly doped to a concentration of $1.8 \times 10^{18} \mathrm{~cm}^{-3}$ and undoped layers as buffers for a consequent HVPE growth of thick layers. The material parameters of both MOCVD 'templates' are summarised in Table I, and a good agreement with previous results reported for similar samples can be seen [6,7].

Table I. Material parameters of both MOVPE-GaN buffer and HVPE-GaN layers.

\begin{tabular}{|c|c|c|c|c|c|c|c|}
\hline & $\begin{array}{l}\mathrm{n}_{\text {Hall }} \\
(300 \mathrm{~K}) \\
\left(\mathrm{cm}^{-3}\right)\end{array}$ & $\begin{array}{l}\mu_{\text {Hall }} \\
\left(\begin{array}{l}300 \mathrm{~K}) \\
\left(\mathrm{cm}^{2} / \mathrm{Vs}\right)\end{array}\right.\end{array}$ & $\begin{array}{l}\omega \\
\mathrm{FWHM} \\
(\mathrm{arcsec})\end{array}$ & $\begin{array}{l}\omega-2 \Theta \\
\mathrm{FWHM} \\
(\operatorname{arcsec})\end{array}$ & $\begin{array}{l}\mathrm{D} \\
(\mu \mathrm{m})\end{array}$ & $\begin{array}{l}\mathrm{DBE} \\
\mathrm{FWHM} \\
(\mathrm{meV})\end{array}$ & $\begin{array}{c}\Delta \mathrm{E}_{\mathrm{DBE}} \\
(\mathrm{meV})\end{array}$ \\
\hline $\begin{array}{c}\text { buffer 1 } \\
(\text { Si doped) }\end{array}$ & $1.8 \times 10^{18}$ & 250 & 273 & 45 & 0.70 & 11.8 & 16.6 \\
\hline $\begin{array}{c}\text { buffer 2 } \\
\text { (undoped) }\end{array}$ & $2 \times 10^{17}$ & 80 & 261 & 36 & 0.77 & 3.6 & 17.3 \\
\hline$\# 1$ (reference) & $3 \times 10^{18}$ & 85 & 725 & 45 & 0.70 & 4.8 & 15.9 \\
\hline $\begin{array}{c}\# 2 \text { (on buffer 1) } \\
\text { measured } \\
\text { corrected }\end{array}$ & $\begin{array}{l}2.3 \times 10^{17} \\
6.2 \times 10^{16}\end{array}$ & 270 & 503 & 59 & 0.58 & 6.8 & 10.8 \\
\hline $\begin{array}{c}\# 3 \text { (on buffer 2) } \\
\text { measured } \\
\text { corrected }\end{array}$ & $\begin{array}{l}3.6 \times 10^{16} \\
2.0 \times 10^{16}\end{array}$ & $\begin{array}{l}515 \\
630\end{array}$ & 227 & 17 & 0.98 & 2.2 & 9.3 \\
\hline
\end{tabular}



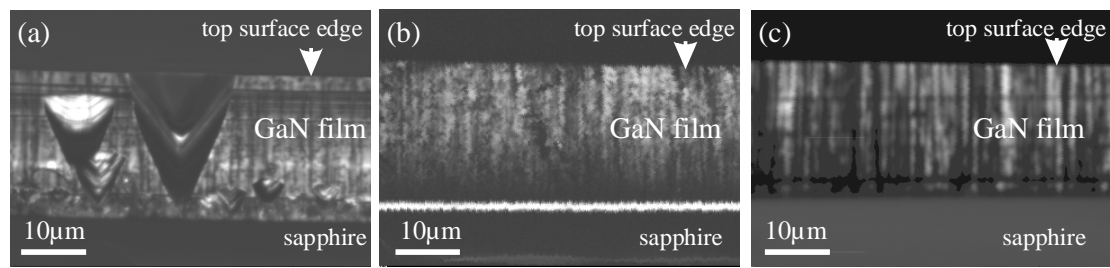

Fig.1 Panchromatic CL images of cross-sections of the GaN layers grown at the same growth conditions on sapphire without a buffer (a); with Si-doped MOCVD-GaN (b); and with undoped MOCVD-GaN 'template' layers (c).

Figure 1 presents CL images of cross-sections of the GaN layers with a comparable thickness of 22-25 $\mu \mathrm{m}$. Fig.1(a) shows a cross-section of the layer grown by conventional nitridation pretreatment of sapphire and Fig.1(b) and (c) show the cross-sections of the layers grown on MOCVD 'templates' intentionally doped with $\mathrm{Si}$ and undoped, respectively. As shown in Fig.1(a) many bright columns can be seen, some of them even protruding to the top of the surface. On the other hand, the cross-sections of the films in Fig.1(b) and (c) are clean and columns do not occur. The spatially resolved CL spectra taken from the bright columnar region show a broad band typical of highly doped material [5,8]. The interface region in sample \#2 appears as a bright stripe with a thickness of $\sim 2$ $\mu \mathrm{m}$ attributed to the Si-doped GaN 'template' with a carrier concentration of $1.8 \times 10^{18} \mathrm{~cm}^{-}$ 3 . The bright columnar region and the columns protruding to the surface in the layer without a buffer were reported [9] to be responsible for the high residual Hall concentration in such layers typically higher than (3-4) $\times 10^{18} \mathrm{~cm}^{-3}$. The removal of the columnar structure in the layers grown on MOCVD 'templates' results in a reduction of the free carrier concentration and an increase of the mobility determined by Hall effect measurements compared to the layers without a buffer. It should be mentioned that the measured carrier concentrations of HVPE layers grown on MOCVD 'templates' in both cases (samples \#2 and \#3) are about one order of magnitude lower than that of the underlying MOCVD layers. Thus there is a need of correction of these values using a twolayer model [10] and the corrected values are shown in Table I. We point out that both Hall-effect measured and corrected values of the mobility are lower than one can expect for the respective values of free carrier concentration, indicating compensation in the films. The latter is also evident from the temperature dependence of the electron concentrations and mobilities shown in Fig.2.
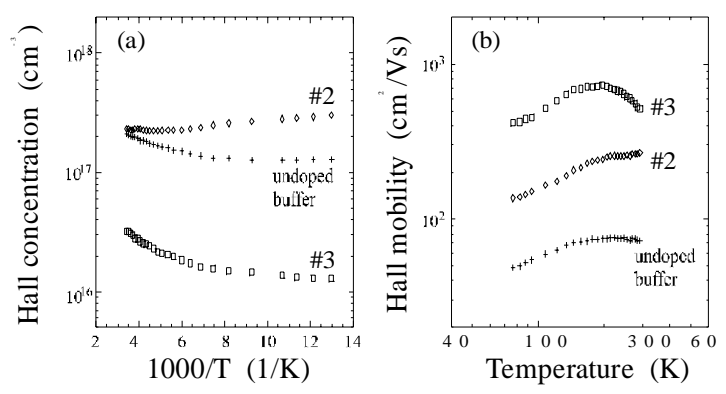

Fig.2 (a) Hall-effect electron concentration vs reciprocal temperature and (b) Hall electron mobility vs temperature. 
The 2K PL spectra of both MOCVD buffers and HVPE layers shown in Fig.3(a) and (b) respectively, exhibit intense near-bandedge emission due to donor-bound excitons (DBE) at about $3.48 \mathrm{eV}$. The FWHM shown in Table I can not be directly correlated with the structural and electrical quality. However, the uniform Si doping in the MOCVD film (buffer 1) results in a clear broadening of the DBE line, and the non-uniform doping of the columnar structure in the reference sample (\#1) results in a broad asymmetric band below the exciton lines. It is interesting to note that while MOCVD-GaN displays a well defined yellow luminescence band around $2.2 \mathrm{eV}$, the HVPE layers do not reproduce that feature and show no luminescence in this region, although a weak red emission around $1.9 \mathrm{eV}$ identified in HVPE GaN layers without a buffer is still present in the PL spectra of layers grown on MOCVD 'templates'. Additionally, the exciton positions $\mathrm{E}_{\mathrm{DBE}}$ are shifted to higher energies with respect to the energetic position of strain-free homoepitaxial grown $\mathrm{GaN}$ (3.471 eV [11]). The shift, $\Delta \mathrm{E}_{\mathrm{DBE}}$ (Table I), is slightly larger for the MOCVD-GaN undoped sample compared to the Si-doped one, due to a higher compressive stress in the layer and consistent with previously reported results [6,7]. The shift is much smaller in HVPE-GaN layers grown on MOCVD buffers, indicative of a presence of a relaxation mechanism in the structure.

The x-ray diffraction measurement was also applied to evaluate the GaN crystalline quality. The strong difference in FWHM values in both $\omega$ and $\omega / 2 \theta$ scans is reflected in the typical elliptic shape of (0002) reciprocal lattice points, which was reported for both MOCVD [12] and HVPE [8] grown layers. This shape is usually explained by mosaicity in the films. A more detailed description of HRXRD results for HVPE layers grown without a buffer can be found elsewhere [8]. The FWHM of the GaN films grown on MOCVD 'templates' were about $500(\# 2)$ and $220 \operatorname{arcsec}(\# 3)$ which is narrower than the 725 arcsec (\#1) obtained for unbuffered growth (Table 1). These results suggest that a reduction in the mosaicity by the growth on MOCVD 'template' buffer is effective to improve the crystalline quality of the films. In addition, it was found that the introduction of Si reduces the size of the coherent scattering areas. The average size of the areas, D, using the Sherrer formula are shown in Table I. This effect was reproduced in the thick HVPE-GaN layers grown on the Si-doped buffer, while the thick GaN films grown on the undoped buffer exhibit a much narrower $\omega / 2 \theta$ line and an increased size of the coherent scattering areas. The difference in the effect of Si-doped and undoped MOCVD buffers on the quality of consequent thick layers may be attributed to the change of the dislocation structure and stress relaxation mechanism in the buffers by the Si doping. In

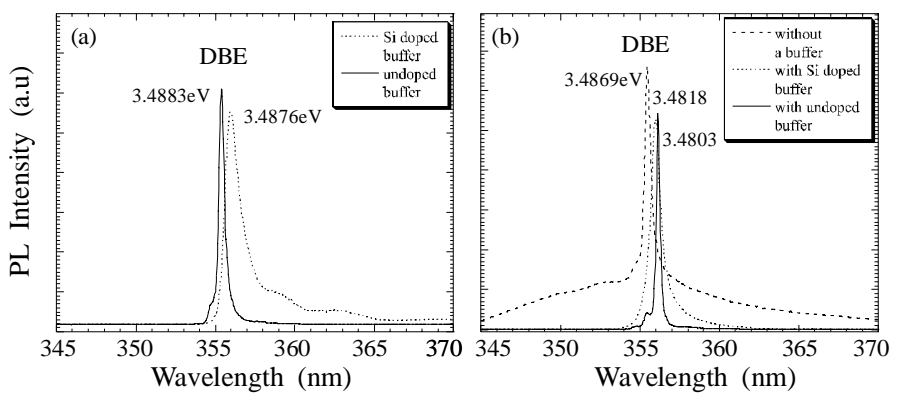

Fig.3 PL spectra in the near-band-gap region of (a) the MOCVD-GaN 'templates and (b) the HVPE-GaN layers. 

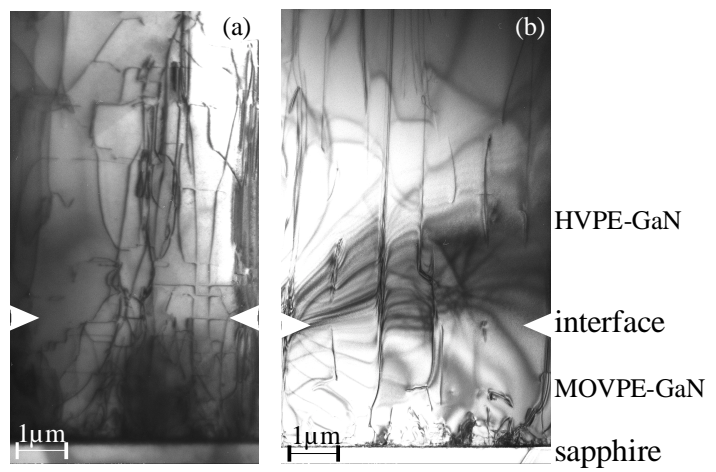

Fig.4 Cross-sectional TEM images of HVPE-GaN/ MOCVD-GaN/sapphire interfaces for layers grown on (a) Si-doped and (b) undoped MOCVD-GaN 'templates'.

order to reveal their defect structure cross-sectional TEM micrographs of the films grown on doped and undoped buffer layers are shown in Fig.4(a) and (b), respectively. The dominant defects are dislocations due to the misfit strain introduced by the lattice mismatch. The doped buffer layer (Fig.4a) reveals dislocation lines oriented irregularly, and a high density of defects parallel to the interface being most probably basal plane dislocations and dislocation loops randomly distributed throughout the whole buffer layer. The overgrown HVPE layer inherits the same character of dislocation distribution, but with a much lower density of $\sim 7 \times 10^{7} \mathrm{~cm}^{-2}$ as a result of promoted interactions and thus annihilation of some of them. The undoped buffer and the overgrown HVPE layer show quite different defect structure (Fig.4b). A $500 \mathrm{~nm}$ region at the MOCVD-GaN/sapphire interface has a high density of stacking faults and half loops and at that thickness straight threading dislocations originate lying close to the [0001] growth direction. Some of them propagate in the overgrown HVPE layer. Others of the same character originate at the interface between the buffer and the overgrown layer. Between dislocations $\left(\sim 4 \times 10^{7} \mathrm{~cm}^{-2}\right)$, good quality regions of about 1-2 $\mu \mathrm{m}$ thickness are seen, keeping an almost constant value throughout the HVPE-GaN film.
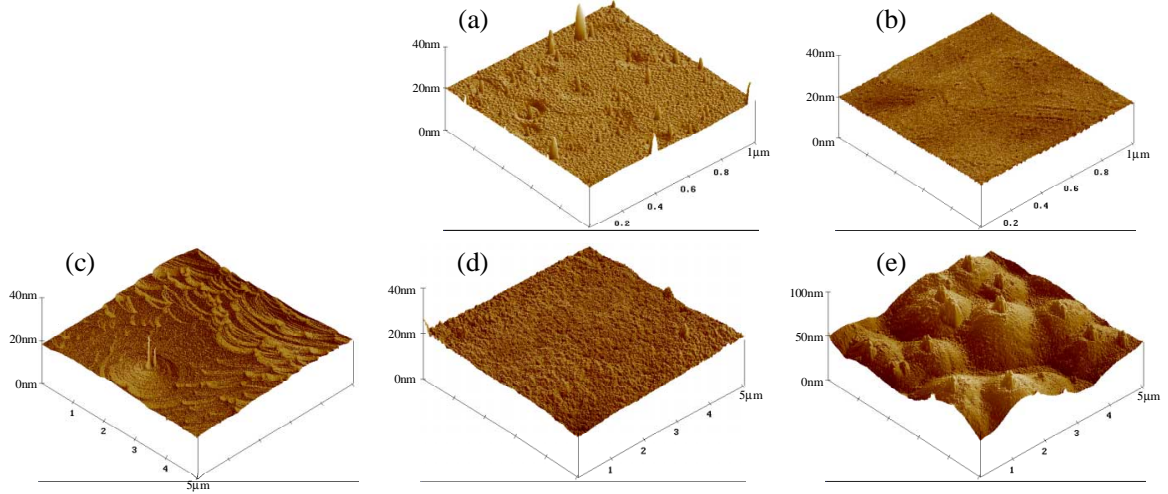

Fig.5 Three-dimensional AFM images of the as-grown top surface of Si-doped MOCVD-GaN (a); undoped MOCVD-GaN (b); HVPE-GaN without a buffer (c); HVPE-GaN on Si-doped MOCVD-GaN 'template' (d); HVPE-GaN on undoped MOCVD-GaN 'template' (e). 
The AFM images shown in Fig.5 reveal the microstructure of the top surfaces of the layers studied. The morphology of the undoped MOCVD 'template' (Fig.5b) appears very smooth with Rms of $\sim 0.65 \mathrm{~nm}$ (from an area of $1 \mathrm{x} 1 \mu \mathrm{m}^{2}$ ), while the surface of Si-doped MOCVD film is slightly rough $(\mathrm{Rms}=1.55 \mathrm{~nm})$ with well defined screw dislocations (Fig.5a). This surface appears to promote nucleation centres for subsequent HVPE growth and leads to a quite smooth microsurface of the consequent HVPE layer (Fig.5d). The reference HVPE sample exhibits a typical (for high growth rate, $\sim 100 \mu \mathrm{m} / \mathrm{h}$ ) growth surface of terraced hillocks mediated by screw dislocations and terrace bunching by edge dislocations (Fig.5c). No terrace bunching is evident in the layers grown on MOCVD buffers (Fig.5d, e) although hillocks with very smooth slopes are present in the HVPE layer grown on undoped buffer (Fig.5e). A change in the dominate growth mechanism is clearly observed in the HVPE-GaN layers using MOCVD buffers, most probably due to change of the structural defect arrangement.

The physical basis for the observation of an optimum buffer layer for physical properties of the main $\mathrm{GaN}$ films remains unclear. We speculate that achieving an optimum buffer is a balance between surface roughness and internal defect structure. Because the buffer with a rough surface evolves specific defects most of them will propagate into the subsequently grown layer. Therefore, the main film grown on a rough buffer layer cannot have high-quality. In contrast, the smooth buffer layer cannot supply a perfect crystallographic template for the main GaN epilayer, therefore the consequently grown GaN film cannot be morphologically perfect although owing better crystal quality.

\section{CONCLUSION}

Good-quality thick GaN layers have been deposited by HVPE on MOCVD-GaN 'template' layers. The defective columnar structure disappeared in the layers. The structural, optical and electrical properties are considerably improved compared to that obtained by growth directly on sapphire. The HVPE homoepitaxial overgrowth on MOCVD 'templates' appeared to be effective in reducing crystalline defects in thick HVPE-GaN films. Additionally, the GaN 'templates' were grown by the well developed and controllable MOCVD technique. Further investigations to clarify the HVPE-MOCVD nucleation mechanism will result in an optimisation of homoepitaxial regrowth.

\section{REFERENCES}

1. R.J. Molnar, W. Götz, L.T. Romano, N.M. Johnson, J. Cryst. Growth, 178, 147 (1997).

2. T. Shibata, H. Sone, K. Yahashi, M. Yamaguchi, K. Hiramatsu, N. Sawaki, N. Itoh, J. Cryst. Growth, 189/190, 67 (1990).

3. I. Akasaki, Mat. Res. Soc. Symp. Proc., 482, 3 (1998).

4. E.J. Tarsa, B. Heying, X.H. Wu, P. Fini, S.P.DenBaars, J.S. Speck, J.Appl.Phys. 82, 5472 (1997).

5. P. Paskova, E.Goldys, B.Monemar, J. Cryst. Growth, 203, 1 (1999).

6. S. Ruvimov, Z. Liliental-Weber, T: Suski, J.W. AgerIII, J. Washburn, J. Krueger, C. Kisielowski, E.R. Weber, H. Amano, I. Akasaki, Appl. Phys. Lett., 69, 990 (1996).

7. N.M. Shmidt, A.V. Lebedev, W.V. Lundin, B.V. Pushnyi, V.V. Ratnikov, T.V. Shubina, A.A. Tsatsul'nikov, A.S. Usikov, G. Pozina, B. Monemar, Mater. Sci. \& Enginer. B, (1999) (in press).

8. T. Paskova, E.M. Goldys, R. Yakimova, E.B. Svedberg, A. Henry, B. Monemar, J. Cryst. Growth, (1999) (in press).

9. B. Arnaudov, T. Paskova, E.M. Goldys, R. Yakimova, S. Evtimova, I.G. Ivanov, A. Henry, B. Monemar, J. Appl. Phys., 85, 7888 (1999).

10. D. Look, R. Molnar, Appl. Phys. Lett. 70, 3377 (1997).

11. K. Kornitzer, T. Ebner, K. Thonke, R. Sauer, C. Kirchner, V. Schwegler, M. Kamp, M.

Leszczynski, I. Grzegory, S. Porowski, Phys. Rev. B 60, 1471 (1999).

F99W3.14 
12. H.Sato, H. Takahashi, A. Watanabe, H. Ota, Appl. Phys. Lett., 68, 3617 (1996). 